\title{
Research on the Properties of Rigid Polyurethane Foam with Heteroaromatic Polyol \\ Hong GUO ${ }^{a}$, Qun GAO ${ }^{b, *}$, Chun-Fa OUYANG ${ }^{c}$
}

\author{
School of Materials Science \& Engineering, Shanghai Institute of Technology, Shanghai, 201418, \\ China

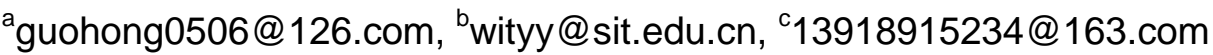 \\ ${ }^{*}$ Corresponding author
}

Keywords: Heteroaromatic Polyol, Rigid Polyurethane Foam, Thermal Stability, Flame Retardancy.

\begin{abstract}
Rigid polyurethane foams (RPUFs) were prepared by heteroaromatic polyol as the matrix polyol, methylphosphonate (DMMP) was added to improve its flame retardancy, and the relative performances were studied. Thermal stability were measured by dynamic mechanical analysis (DMA) and thermo gravimetric analysis (TG), limiting oxygen index (LOI) was selected to investigate the flame retardancy. Results showed that the glass transition temperature (Tg) of RPUF prepared by heteroaromatic polyol was $180^{\circ} \mathrm{C}$ and the initial decomposition temperature was reached to $200^{\circ} \mathrm{C}$, much higher than that of RPUFs prepared by ordinary polyether and polyester polyol, demonstrated that the special heteroaromatic polyol exhibited a better thermal stability. In addition, the characterization of flame-retardant RPUFs displayed that the LOI value increased significantly with the increasing amount of flame retardant and reached to $31.5 \%$ in the experimental range, which illustrated that the flame retardancy was improved apparently. Better char-forming ability was observed from TG test. The char residue was up to $34 \%$ at $800{ }^{\circ} \mathrm{C}$ when the flame retardant additive was $20 \%$. Scanning electron microscope (SEM) image proved that a compact char-layer covered on the surface of the material after burning. The compressive strength was $0.14 \mathrm{MPa}$ when the addition amount was $25 \%$, which indicated that the addition of DMMP has little effect on the compressive strength of RPUF with heteroaromatic polyol.
\end{abstract}

\section{Introduction}

Rigid polyurethane foam (RPUF) attracts a wide attention and develops rapidly in recent years for its excellent properties, such as low density, high abrasion resistance and excellent mechanical properties[1]. Especially, it has excellent insulation properties because of its inferior thermal conductivity, and is mainly used as insulation materials in the construction industry and pipeline equipment. However, the LOI value of RPUF without flame retardant is only 16-18\%, seriously threat to the safety because of toxic smoke releasing when exposed to fire[2-3], and restrict its application immensely. Hence, it is of great importance to improve the flame retardancy for its popularization[4-5].

Generally, additive and reactive flame retardant was used to modify the flame retardancy of RPUF. Additive flame retardant was added into the matrix resin in the physical way as it has no reactivity. It is easy to operate and has better flame retardant effect, so it is the most widely used method in flame retardancy of RPUF. However, the defects of additive flame retardants can't be ignored, such as easy leaching, poor compatibility and reduction of mechanical properties[6]. Compared with additive flame retardant, the reactive flame retardant introduces the flame retardant elements into polymer chains through chemical reaction, improves the stability of flame-retardant RPUF and has little effect on the mechanical properties[7].

In addition to the two methods of flame retardancy above, the structure modification is also an important method of flame retardant. Generally, parts of the polymer are replaced by groups with higher thermal stability, such as heteroaromatic, aromatic and isocyanate ring structure. Masiulanis et al[8] introduced the heteroaromatic structure into the main chain of polyurethane, the thermal stability was obviously improved on the basis of comprehensive performance unchanged, and the 
thermal degradation temperature can reach to $500{ }^{\circ} \mathrm{C}$. The thermal stability of RPUF is improved by raising the decomposition temperature of the polymer itself. Therefore, the addition of polyols with special structure has become an important aspect of the flame-retardant RPUF.

In this work, a special heteroaromatic polyol was selected as the matrix resin to improve the thermal stability, besides, methylphosphonate (DMMP) was added to imprve the flame retardancy of RPUF. Thermal stability, dynamic mechanical performance and mechanical property of flame retardant RPUFs were studied.

\section{Experiment}

\section{Materials}

Methane diphenyl diisocyanate (MDI) is produced by Huntsman Corporation, whose commercial designation is 5005 with average functionality 2.6-2.7, and characterized by $\mathrm{NCO} \%=30.75$, viscosity to $25^{\circ} \mathrm{C}=220 \mathrm{mPa} \cdot \mathrm{s}$.

Polyol-1 $(60 \mathrm{wt} \%)$ is a kind of heteroaromatic polyether polyol based on isocyanurate with end group -OH. The hydroxyl value is $250-320 \mathrm{mgKOH} \mathrm{g}^{-1}$, functionality is 3 .

Polyol-2 (60wt\%) is a kind of polyester polyol based on isocyanurate with end group $-\mathrm{OH}$. The hydroxyl value is $300-400 \mathrm{mgKOH} \mathrm{g}^{-1}$.

Polyol-3 (30wt\%) is a kind of sucrose polyether polyol, whose hydroxyl value is $500 \mathrm{mgKOH} \mathrm{g}^{-1}$, the functionality is 5 . Its viscosity to $25^{\circ} \mathrm{C}$ is $5800 \mathrm{mPa} \cdot \mathrm{s}$ and commercial grade designation is 635 .

Polyol-4 (30wt\%) is a variety of sucrose polyether polyol with hydroxyl value of $380 \mathrm{mgKOH} \mathrm{g}{ }^{-1}$. Its functionality is 6 , the viscosity to $25^{\circ} \mathrm{C}$ is $11250 \mathrm{mPa} \cdot \mathrm{s}$ and commercial grade designation is 8490.

DMMP was used as flame retardant, but wasn't used in the original formula.

Triethylenediamine and stannous octoate were used as catalysts.

HCFC-141b (dichlorofluoroethane) was used as blowing agents and polysilicone was used as foam stabilizer.

\section{Preparation of Samples}

The foams were prepared by hand mixing technique. Mixed the polyol with catalysts, blowing agent and surfactant first, then the flame retardant was added into the mixture with different proportion $(0,5,10,15,20,25 \mathrm{wt} \%)$ and stirred well. Finally, the isocyanate was added to the formulated polyol and mixed for 10s, standing and automatic foaming. After the preparation, the foams were kept in an oven at $70^{\circ} \mathrm{C}$ for $24 \mathrm{~h}$ to complete the polymerization reaction. The parameters of RPUFs prepared by varieties of polyols were shown in Tab.1

Tab.1 Parameters of different RPUFs

\begin{tabular}{cccc}
\hline Materials & Sample 1[pbw] & Sample 2[pbw] & Sample 3[pbw] \\
\hline Polyol-1 & 60 & -- & -- \\
Polyol-2 & -- & 60 & - \\
Polyol-3 & -- & -- & 30 \\
Polyol-4 & -- & -- & 30 \\
triethylenediamine & 0.8 & 0.8 & 0.8 \\
stannous octoate & 0.2 & 0.2 & 0.2 \\
polysilicone & 1.0 & 1.0 & 1.0 \\
HCFC-141b & 23.0 & 23.0 & 23.0 \\
Flame retardant & 15.0 & 15.0 & 15.0 \\
MDI & 100 & 100 & 100 \\
\hline
\end{tabular}

Sample 1 was the RPUF prepared by heteroaromatic polyether polyol;

Sample 2 was the RPUF prepared by polyester polyol;

Sample 3 was the RPUF prepared by polyether polyol. 


\section{Characterization}

Thermo gravimetric analysis (TG) was performed on a TG analyzer (NETZSCH, Germany) from 30 to $800^{\circ} \mathrm{C}$ at a heating rate of $10^{\circ} \mathrm{C} \mathrm{min}{ }^{-1}$ under a dynamic nitrogen flow of $30 \mathrm{ml} \mathrm{min}^{-1}$.

Dynamic mechanical analysis (DMA) was performed using DMA apparatus (NETZSCH, Germany), which was carried out from room temperature to $200^{\circ} \mathrm{C}$ at a heating rate of $3^{\circ} \mathrm{C} \mathrm{min}{ }^{-1}$ at $1 \mathrm{~Hz}$.

Limiting oxygen index (LOI) was used to characterize the flame retardant of RPUF which according to GB/T 2406-2009, and the size of the specimen was $150 \times 10 \times 10 \mathrm{~mm}^{3}$ (length $\times$ width $\times$ thickness).

Scanning electron microscope (SEM) was performed to investigate the morphology of solid residues collected at the end of LOI after being gold-sputtered, and the accelerating voltage was $20 \mathrm{kV}$.

The mechanical properties of the samples were evaluated at $23^{\circ} \mathrm{C}$ using universal testing machine (GALDABINI Corp., Italy). Compression strength is the compressive stress of $10 \%$ relative deformation according to the GB 8813-1988.

\section{Results and Discussion}

\section{Influence of Heteroaromatic Structure on Thermal Stability of RPUF}

The thermal stability of RPUF can be significantly improved by incorporating the organic heteroaromatic groups, such as isocyanurate, polyimide, et al, into the main chain, because of its larger cohesive energy and higher thermal decomposition temperature. These special structures can hinder the movement of the molecular chain, so the thermal stability of polymers can be improved apparently. A kind of heteroaromatic polyol was selected as matrix resin to prepare RPUF in this study, and was compared with ordinary polyether and polyester polyol without special structures, in order to verify the influence of heteroaromatic structure on the properties of RPUF.

Glass transition temperature ( $\mathrm{Tg}$ ) is an important characterization method to illustrate the thermal stability of RPUF, and the peak value of loss tangent $(\tan \delta)$ is the most prevalent criterion of the $\mathrm{Tg}$, so it was used to characterize the thermal stability of different RPUFs and the results were shown in Fig.1.

As observed in Fig.1, the Tg of RPUF prepared by heteroaromatic polyether was $180^{\circ} \mathrm{C}$, while it was $170^{\circ} \mathrm{C}$ for which prepared by ordinary polyester and $165^{\circ} \mathrm{C}$ for which prepared by ordinary polyether. So the thermal stability of RPUF prepared with heteroaromatic polyol was significantly better than that with ordinary polyether and polyester polyol. Moreover, the heat resistance of RPUF with polyester polyol was higher than that with polyether polyol as well.

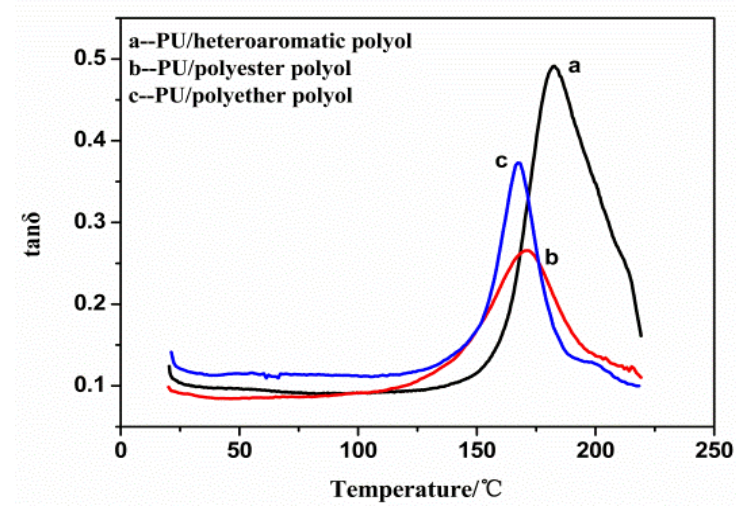

Fig. 1 Tan $\delta$ as a function of temperature for RPUFs

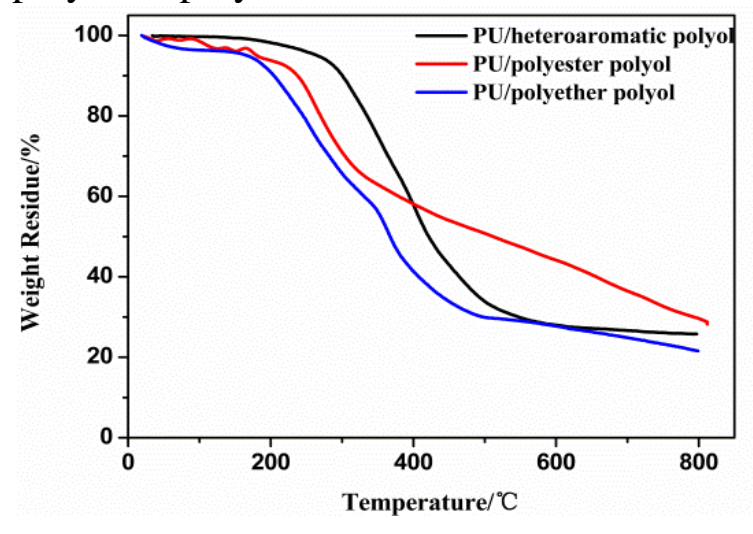

Fig.2 TG curves of different RPUFs

TG curves of three different kinds of RPUFs were shown in Fig.2, as depicted in the figure, the initial decomposition temperature of RPUF with heteroaromatic polyol was $200{ }^{\circ} \mathrm{C}$, higher than that of RPUFs with ordinary polyether and polyester polyol apparently. Especially, it was $40{ }^{\circ} \mathrm{C}$ higher 
than that with polyether polyol, and it was in accordance with the tendency observed from the $\mathrm{Tg}$, which further illustrated that heteroaromatic polyol can improve the thermal stability of RPUF.

Bond strength was the most important factor to affect the thermal stabilities of polymers, and the resonance energy of heteroaromatic structure is much higher than the others because of its conjugated effect. Thus the incorporation of heteroaromatic structure can improve the thermal stability of materials, the thermal stability of RPUF with heteroaromatic polyol was much better than that of RPUF with ordinary polyester and polyether polyol. Additionally, the intermolecular force of polyester polyol is larger than polyether polyol because its carbonyl has a large polarity, and the hydrogen on the carbon atom of ether group is susceptible to oxidation, so the $\mathrm{Tg}$ and initial decomposition temperature of RPUF with polyester polyol was higher than that of RPUF with polyether polyol, illustrating that its thermal stability is better than the RPUF with polyether polyol.

In summary, the thermal stability of RPUF can be improved by heteroaromatic polyol apparently. However, the RPUF without any flame-retardant treatments is flammable, and its application is limited greatly. So the flame retardancy of RPUFs needs to be improved before its application.

\section{Effect of Flame Retardant Additive on Flame Retardancy of RPUF}

Methylphosphonate (DMMP) is an additive flame retardant with high phosphorus content and used in flame-retardant RPUF commonly. In this paper, different addition amount of DMMP $(0,5$, $10,15,20,25 \mathrm{wt} \%$ ) was added into the RPUF, and the effect on the performance of RPUF was studied. TG curves of RPUFs modified with different amount DMMP was shown in Fig.3.

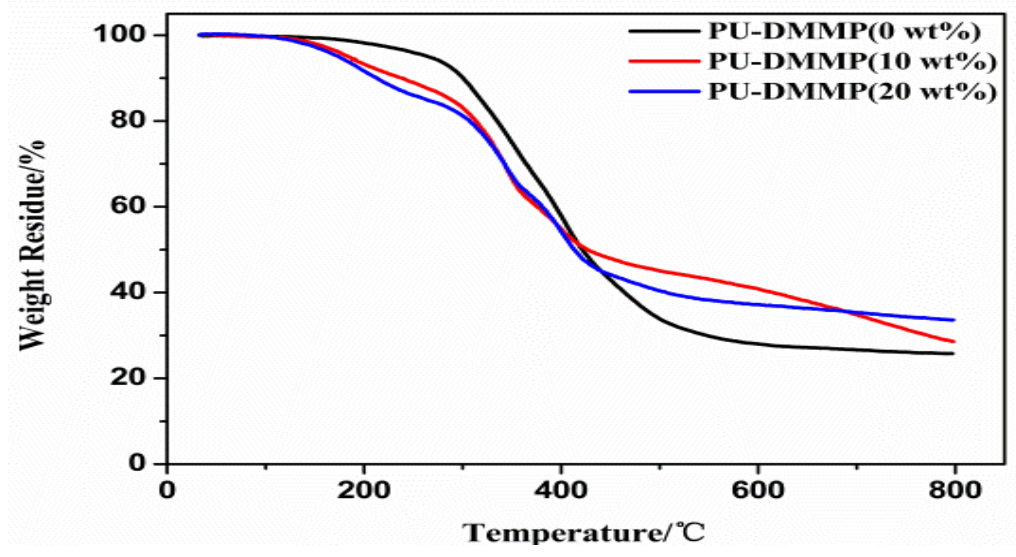

Fig.3 TG curves of flame-retardant RPUFs

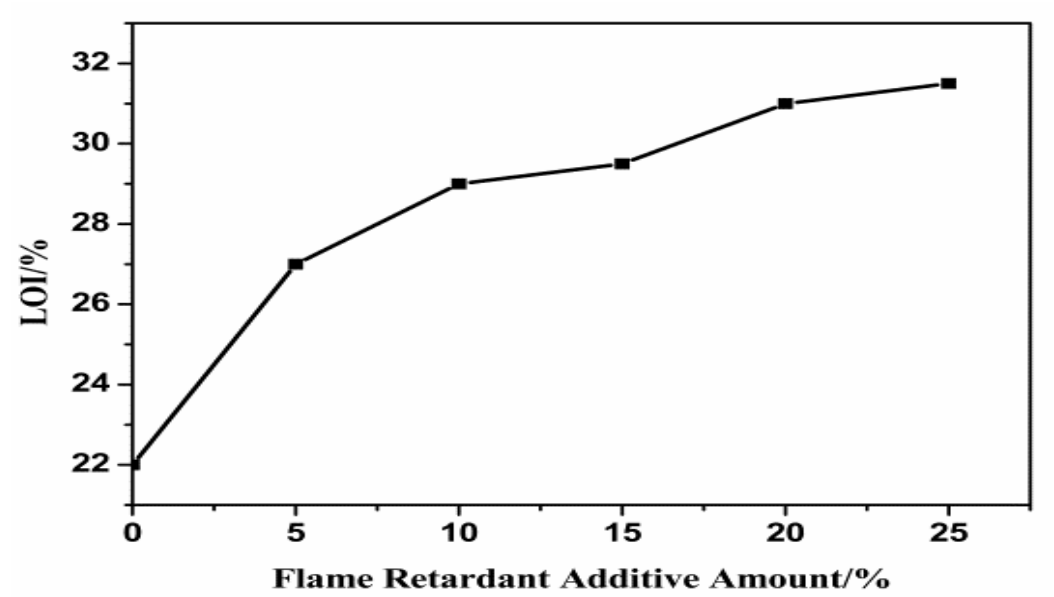

Fig.4 Effect of DMMP concentration on LOI of flame-retardant RPUFs

As observed in Fig.3, the initial decomposition temperature of flame-retardant RPUFs was lower than that of RPUF without flame retardant and decreased with the increasing amount of DMMP. In 
addition, the initial decomposition temperature was $180^{\circ} \mathrm{C}$ and was in accordance with the decomposition temperature of DMMP; moreover, the weight loss of the first stage was identical with the addition amount of the flame retardant. The char residue of flame-retardant RPUF can reach $34 \%$ when the addition amount of DMMP was $20 \%$ and was $28 \%$ when the addition amount was $10 \%$, both higher than that of RPUF without DMMP, illustrating that the char residue increased with the increasing amount of DMMP.

The LOI of RPUFs with different DMMP content was tested and the results were given in Fig.4. The LOI values of RPUFs raised with the increase of DMMP content, and reached a maximum of $31.5 \%$ when the dosage was $25 \%$ in the experimental range, while that of RPUF without flame retardant was only $22 \%$. The LOI increased significantly and greatly improved the flame retardancy.

The charring mechanism of the condensed phase can be used to explain the trend of TG and LOI of flame-retardant RPUFs. DMMP decomposed first to generate phosphoric acid and metaphosphoric acid when it was heated, then the products promoted the char-forming of the polymer and generated char-layer covered on the surface of the polymer. The feedback of heat and combustible gas to the matrix was reduced, and then the flame retardancy of RPUF was improved. As depicted in Fig.5, a dense char-layer was observed on the surface of flame-retardant RPUF after burning, preventing the transmitting of combustion and heat to the polymer in the combustion process.

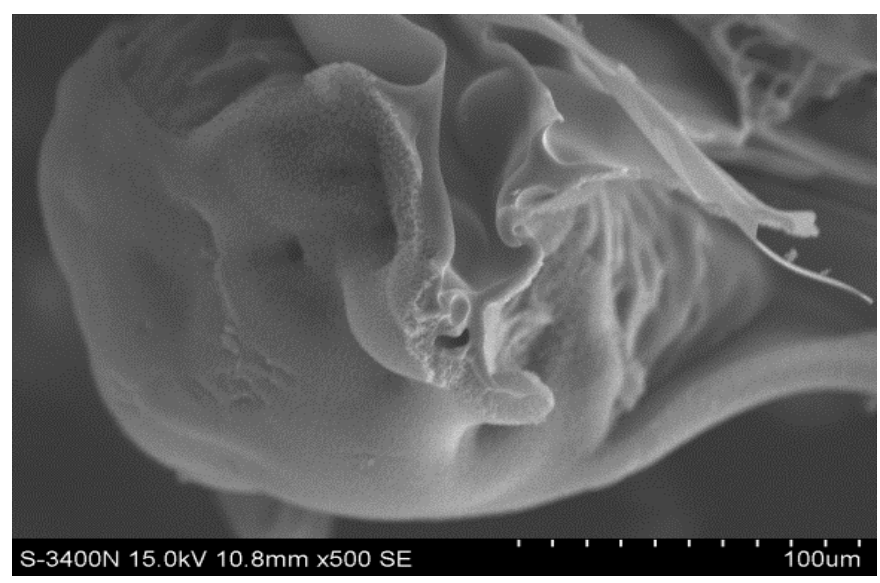

Fig.5 SEM image of flame-retardant RPUF

\section{Effect of Flame Retardant Additive on Mechanical Property of RPUF}

The flame retardancy of RPUF was significantly improved with the increasing amount of the flame retardant, but the addition of flame retardant affected its mechanical property to some extent. Fig. 6 showed the compressive strength curves with the addition amount of the flame retardant.

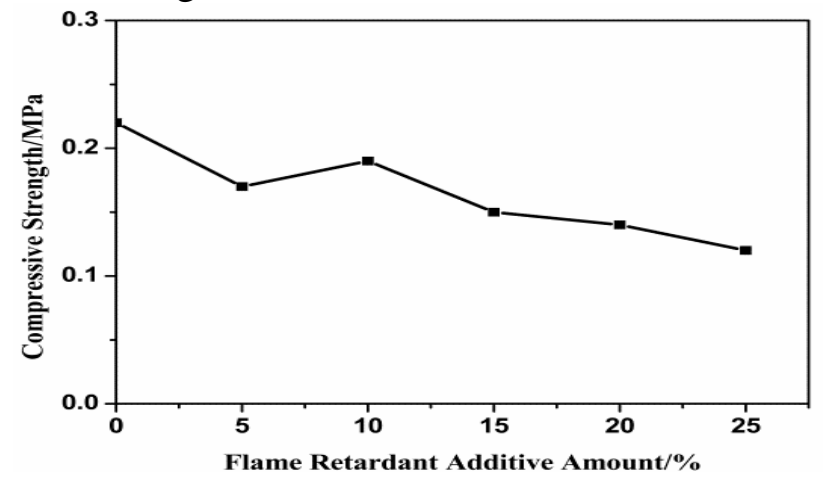

Fig.6 Compressive strength of flame-retardant RPUFs

As was seen from Fig.6, the compressive strength of flame-retardant RPUFs decreased little with the increasing amount of the flame retardant. It was $0.22 \mathrm{MPa}$ for the RPUF without flame retardant 
and reduced to $0.14 \mathrm{Mpa}$ when the flame retardant addition increased to $25 \%$. This is mainly because the flame retardant couldn't distribute equally in the RPUF, so many broken foams were generated and then the compressive strength was reduced. In addition, DMMP played a plasticized role in the synthesis of RPUF to some extent, reduced the mechanical properties of RPUF itself. Hence, the compressive strength decreased with the increase of flame retardant. However, the special heteroaromatic structure of the polyol could prevent the reduction of the compressive strength, thus, it still conform to the standard even at $25 \%$ dosage.

\section{Conclusion}

The Tg of RPUF prepared by heteroaromatic polyol was $180^{\circ} \mathrm{C}$, the initial decomposition temperature was reached to $200^{\circ} \mathrm{C}$, much higher than that prepared by ordinary polyether and polyester polyol, demonstrated that the special heteroaromatic polyol has a better thermal stability. In addition, the LOI value increased significantly with the increasing amount of DMMP, and reached to $31.5 \%$ in the experimental range, improved the flame retardancy significantly. Finally, better char-forming ability was observed from TG test, and the char residue was up to $34 \%$ at 800 ${ }^{\circ} \mathrm{C}$ when the flame retardant additive was $20 \%$. SEM image proved that a compact char-layer covered on the surface of the material after burning.

\section{Reference}

[1]D. W. Hatchett, G. Kodippili, J. M. Kinyanjui, F. Benincasa, L. Sapochak. Polym. Degrad. Stab., 87(2005), 555-561.

[2]N. Usta. Investigation of fire behavior of rigid polyurethane foams containing fly ash and intumescent flame retardant by using a cone calorimeter. J. Appl. Polym. Sci., 124(2012), 3372-3382.

[3]H. Singh, A. K. Jain. Ignition, combustion, toxicity, and fire retardancy of polyurethane foams: A comprehensive review. J. Appl. Polym. Sci., 111(2009), 1115-1143.

[4]H. Singh, A. K. Jain, T. P. Sharma. Effect of phosphorus-nitrogen additives on fire retardancy of rigid polyurethane foams. J. Appl. Polym. Sci., 109(2008), 2718-2728.

[5]G. J. Wang, W. Xu. Influence of caged bicyclic phosphate and $\mathrm{CaCO}_{3}$ nanoparticleson char-forming property of PU rigid foams. Polym Degrad Stab., 98(2013), 2323-2330.

[6]D. Q. Hoang, J. Kim, B. N. Jang. Synthesis and performance of cyclic phosphorus-containing flame retadants. Polym Degrad Stabil., 93(2008), 2042-2047.

[7]N. I. Yanchuk. Organic solvents as catalysts of formation of phosphorus-containing thiosemi carbazides. Russian Journal of General Chemistry., 76(2006), 1236-1239.

[8]B. Masiulanis, R Zielinski. Mechanical, thermal and electric properties of polyurethaneimide elastomers. J. Appl. Polym. Sci., 30(2003),2731-2741. 